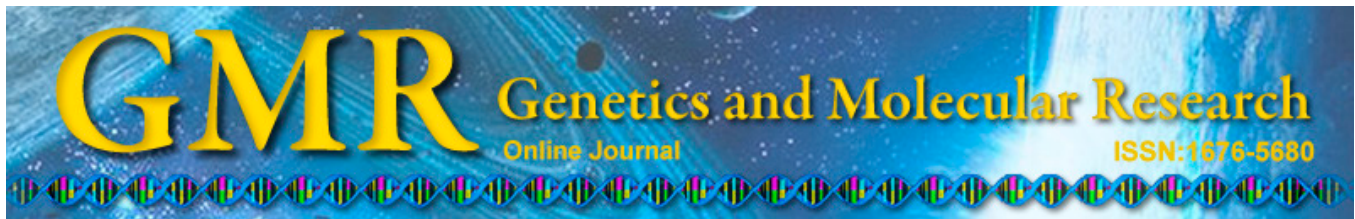

\title{
Increased serum ADAMTS-4 in knee osteoarthritis: a potential indicator for the diagnosis of osteoarthritis in early stages
}

\author{
W. Li, C. Du, H. Wang and C. Zhang \\ Department of Nuclear Medicine, \\ Qianfoshan Hospital Affiliated to Shandong University, Jinan, China \\ Corresponding author: C. Zhang \\ E-mail: liwenqas@126.com
}

Genet. Mol. Res. 13 (4): 9642-9649 (2014)

Received January 13, 2014

Accepted September 6, 2014

Published November 14, 2014

DOI http://dx.doi.org/10.4238/2014.November.14.9

\begin{abstract}
We compared serum levels of a disintegrin and metalloproteinase with thrombospondin motifs (ADAMTS)-4, ADAMTS-5, matrix metalloproteinase (MMP)-1, and MMP-3 in patients with different stages of knee osteoarthritis (OA), and investigated the clinical significance of diagnosing OA in early stages. OA patients were divided into 2 groups: early OA group (44 cases), intermediate and advanced OA group (26 cases). The healthy control group included 30 samples. ADAMTS-4, ADAMTS-5, MMP-1, and MMP-3 levels in the serum were tested using an enzyme-linked immunosorbent assay. A protein-protein interaction network was constructed by seeding the significantly expressed marker, followed by Gene Ontology enrichment analyses using Database for Annotation, Visualization and Integrated Discovery. ADAMTS-4 levels were significantly higher in patients at early stages of OA compared to intermediate or advanced OA and healthy controls. ADAMTS-5, MMP-1, and MMP-3 levels in intermediate and advanced-stage OA patients were significantly higher than those in early-stage OA patients and healthy controls. The proteinprotein interaction network showed that ADAMTS-4 participates in
\end{abstract}


67 interactions. Gene Ontology enrichment analysis validated that genes associated with ADAMTS-4 participate in collagen metabolism and OA. ADAMTS-4 is a potential biomarker as an early diagnostic indicator of OA.

Key words: ADAMTS; MMP; Osteoarthritis

\section{INTRODUCTION}

Osteoarthritis (OA) is a progressive disease of the joints characterized by degradation of articular cartilage (Verma and Dalal, 2011) and is the most common form of arthritis worldwide (Zeggini et al., 2012). The incidence of OA is increasing because of the aging population and the obesity epidemic (Bijlsma et al., 2011). Various risk factors contribute to OA, including smoking, genetics, and mechanical factors, among others (Wei et al., 2013). Pain and loss of function are the dominant symptoms in OA (Arendt-Nielsen et al., 2010; Bijlsma et al., 2011). OA is associated with cartilage destruction, subchondral bone remodeling, and inflammation of the synovial membrane. However, the etiology and pathogenesis underlying this disease are poorly understood (Kapoor et al., 2011).

OA is diagnosed using X-ray, computed tomography, magnetic resonance (MR), and similar imaging techniques. However, these methods cannot examine OA before the changes in the arthrosis profile occur. In addition, biochemical changes occur during early stages. Changes in cartilage metabolism in arthritis are reflected by altered serum and synovial fluid levels (Poole et al., 1994). Thus, serologic examination is a feasible method for diagnosing OA in early stages.

Matrix metalloproteinases (MMPs) are expressed in the joint tissues of patients with OA (Yoshihara et al., 2000). It has been reported that MMP-3 is downregulated by the effect of hyaluronan during early development of OA (Takahashi et al., 1999). MMP-3 activation plays a key role in the development of OA (Lin et al., 2004). A disintegrin and metalloproteinase with thrombospondin motifs (ADAMTS) are a group of proteases found both in mammals and invertebrates (Porter et al., 2005). ADAMTS-4 and ADAMTS-5 efficiently cleave aggrecan at a major cleavage site of the protein backbone of aggrecan between amino acids 373 and 374 (Glasson et al., 2004). ADAMTS-4 and ADAMTS-5 are thought to play an important role in inflammatory joint diseases and cartilage degradation (Mizui et al., 2000; Ameye and Young, 2006).

In this study, we examined the levels of ADAMTS-4, ADAMTS-5, MMP-1, and MMP-3 in the serum of patients with OA and analyzed the correlation between these protein levels and the severity of OA. Our results provide information that will assist in the early diagnosis of OA.

\section{MATERIAL AND METHODS}

\section{Patients}

This study was approved by the research Ethics Committee of our hospital and was performed in accordance with the ethical standards stated in the 1964 Declaration of Helsinki. Written informed consent was obtained from all patients and healthy volunteers prior to their participation in this study.

The present study included 70 patients diagnosed with OA and 30 healthy controls. 
Patients were selected at the Qianfoshan Hospital affiliated to Shandong University, China, between June 2008 and December 2009. Patients were examined by MR (Artoscan 0.2T; Esaote, Italy). Diagnosis criteria of OA were defined by the Sciences Branch of the Chinese Medical Association in 2007.

Participants who met the following criteria were excluded from the study: 1) age $>60$ years; 2) histories of glucocorticoid and/or sterol hormones; 3) two knees showed different grades of OA; 4) had received bilateral knee replacements; 5) other forms of arthritis, or other chronic disease (cancer, diabetes); 6) pregnant and lactating women.

\section{Radiographic assessment of OA}

Radiographic severity was assessed according to the Recht et al. (1993) grading system, which is widely used in Chinese hospitals. Grading of radiographs was performed by 2 experienced observers who were blinded to the patient information. We divided patients into 2 groups: early OA patients, defined as having a radiographic OA of grade 1 or 2, and intermediate and advanced OA patients, defined as having a radiographic OA of grade 3 or 4 .

\section{Measurement of ADAMTS-4, ADAMTS-5, MMP-1, and MMP-3}

Venous blood samples were collected from all subjects after overnight fasting. Serum ADAMTS-4, ADAMTS-5, MMP-1, and MMP-3 concentrations were determined using a commercially available enzyme-linked immuosorbent assay kit (Sigma-Aldrich; St. Louis, MO, USA). Optical density (OD) values at $405 \mathrm{~nm}$ were recorded and were used to compare ADAMTS-4, ADAMTS-5, MMP-1, and MMP-3 levels between patients and controls.

\section{Protein-protein interaction network construction}

To further investigate the functions of significantly expressed markers, we constructed protein-protein interaction (PPI) networks. PPI data were downloaded from STRING (http:// string.embl.de/). The networks were identified using the Cytoscape software.

\section{Functional enrichment analysis}

To analyze the functions of related genes identified in the PPI, we performed Gene Ontology (GO) enrichment analysis and diseases analysis using the Database for Annotation, Visualization and Integrated Discovery (DAVID) (http://david.abcc.ncifcrf.gov/home.jsp) (Huang da et al., 2009).

\section{Statistical analysis}

One-way analysis of variance was utilized to compare differences in ADAMTS-4, ADAMTS-5, MMP-1, and MMP-3 levels using OD in the serum of patients and controls. Results are reported as means $\pm \mathrm{SD}$ and as percentages. Data analyses were performed using SPSS 11.5 for Windows (SPSS, Inc.; Chicago, IL, USA). Differences between groups were considered to be significant when $\mathrm{P}<0.05$. 


\section{RESULTS}

\section{ADAMTS-4 and ADAMTS-5 levels in the serum}

ADAMTS-4 levels in patients and healthy controls are presented in Figure 1A. ADAMTS-4 levels in early OA patients were significantly higher than those in intermediate and advanced OA patients and healthy controls $(\mathrm{P}<0.01)$.

ADAMTS-5 levels in patients and healthy controls are presented in Figure 1B. ADAMTS-5 levels in intermediate and advanced OA patients were significantly higher than those in early OA patients and healthy controls $(\mathrm{P}<0.01)$.
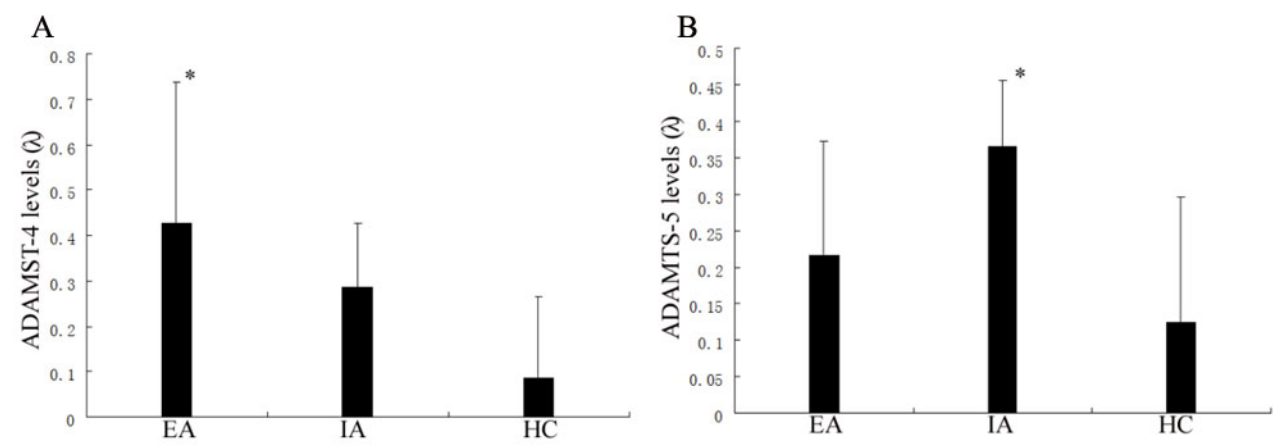

Figure 1. ADAMTS-4 levels (A) and ADAMTS-5 levels (B) in the serum of early osteoarthritis (OA) patients (EA), intermediate and advanced OA patients (IA), and healthy controls (HC).

\section{MMP-1 and MMP-3 levels in the serum}

MMP-1 levels in patients and healthy controls are presented in Figure 2A. MMP-1 levels in intermediate and advanced OA patients were significantly higher than those in early OA patients and healthy controls $(\mathrm{P}<0.01)$.

MMP-3 levels in patients and healthy controls are presented in Figure 2B. MMP-3 levels in intermediate and advanced OA patients were also significantly higher than in early OA patients and healthy controls $(\mathrm{P}<0.05)$.
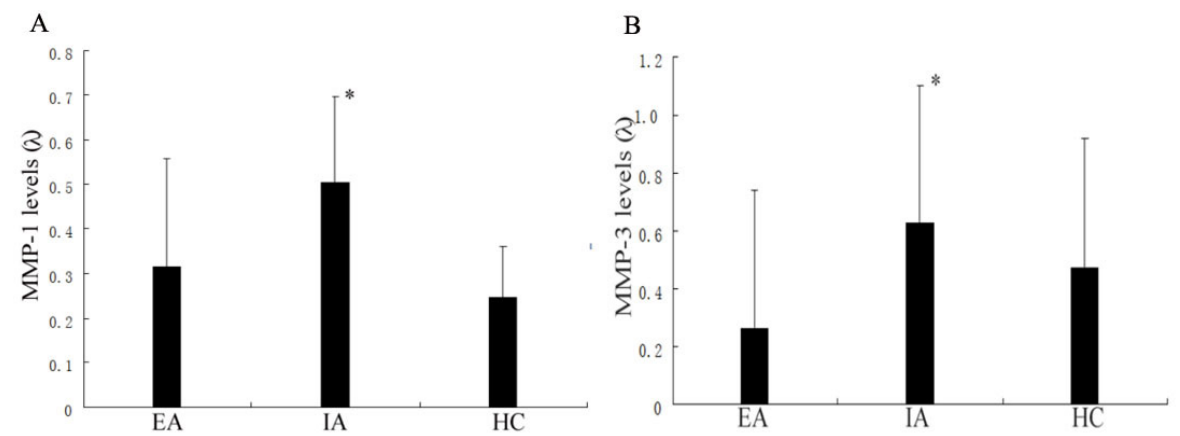

Figure 2. MMP-1 levels (A) and MMP-3 levels (B) in the serum of early osteoarthritis (OA) patients (EA), intermediate and advanced OA patients (IA), and healthy controls (HC). 


\section{Interaction network of the ADAMTS-4}

We constructed a PPI network for ADAMTS-4, the level of which was significantly higher in early OA patients. The PPI network revealed that ADAMTS-4 participates in 67 interactions (Figure 3).

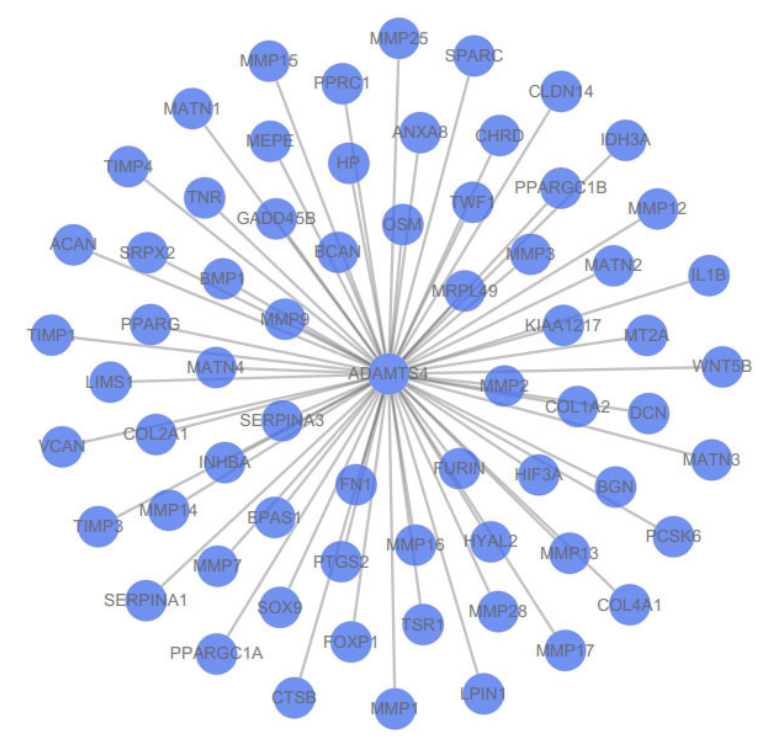

Figure 3. Protein-protein interaction network involving ADAMTS-4.

\section{Functional enrichment analysis}

GO enrichment analyses validated that genes associated with ADAMTS-4 mainly participated in biological process related to collagen metabolism and OA (Table 1). Disease enrichment analysis indicated that several genes are associated with rheumatoid arthritis and OA (Table 2).

Table 1. Top 8 enriched GO terms of biological processes from genes in protein-protein interaction.

\begin{tabular}{|c|c|c|c|}
\hline GO ID & Terms & $P$ value & Genes \\
\hline GO:0030574 & Collagen catabolic process & $2.67 \mathrm{E}-10$ & $\begin{array}{l}\text { MMP9, MMP7, MMP16, MMP3, } \\
\text { MMP13, MMP2, MMP1 }\end{array}$ \\
\hline GO:0044243 & Multicellular organismal catabolic process & $1.55 \mathrm{E}-09$ & $\begin{array}{l}\text { MMP9, MMP7, MMP16, MMP3, } \\
\text { MMP13, MMP2, MMP1 }\end{array}$ \\
\hline GO:0032963 & Collagen metabolic process & $2.52 \mathrm{E}-09$ & $\begin{array}{l}\text { MMP9, MMP7, MMP16, MMP3, } \\
\text { MMP13, MMP2, MMP1 }\end{array}$ \\
\hline GO:0044259 & Multicellular organismal macromolecule metabolic process & 4.87E-09 & $\begin{array}{l}\text { MMP9, MMP7, MMP16, MMP3, } \\
\text { MMP13, MMP2, MMP1 }\end{array}$ \\
\hline GO:0044236 & Multicellular organismal metabolic process & $1.51 \mathrm{E}-08$ & $\begin{array}{l}\text { MMP9, MMP7, MMP16, MMP3, } \\
\text { MMP13, MMP2, MMP1 }\end{array}$ \\
\hline GO:0001501 & Skeletal system development & $6.45 \mathrm{E}-13$ & $\begin{array}{l}\text { MATN3, BMP1, PTGS2, MMP9, COL2A1, } \\
\text { SPARC, MEPE, SOX9, MMP14, } \\
\text { MMP2, MMP13, INHBA, COL1A2, ACAN, } \\
\text { KIAA1217, CHRD, ADAMTS4 }\end{array}$ \\
\hline GO:0060348 & Bone development & 8.33E-08 & $\begin{array}{l}\text { BMP1, PTGS2, COL2A1, SPARC, SOX9, } \\
\text { MMP14, MMP13, MMP2, CHRD }\end{array}$ \\
\hline GO:0001503 & Ossification & $8.92 \mathrm{E}-07$ & $\begin{array}{l}\text { BMP1, PTGS2, COL2A1, SPARC, } \\
\text { MMP14,MMP13, MMP2, CHRD }\end{array}$ \\
\hline
\end{tabular}


Table 2. Top 8 enriched diseases terms from genes in protein-protein interaction.

\begin{tabular}{lcl}
\hline Diseases & P value & Genes \\
\hline Breast cancer & $3.28 \mathrm{E}-05$ & $\begin{array}{l}\text { PTGS2, MMP9, PPARG, HP, MMP3, MMP2, TIMP3, MMP13, } \\
\text { PPARGC1A, MMP1, MMP12, PPARGC1B, IL1B }\end{array}$ \\
Rheumatoid arthritis & $2.92 \mathrm{E}-06$ & $\begin{array}{l}\text { PTGS2, MMP9, MMP7, IL1B, SERPINA1, COL2A1, MMP3, MMP13, } \\
\text { MMP2, MMP12, MMP1 }\end{array}$ \\
Abdominal aortic aneurysm & $6.63 \mathrm{E}-09$ & MMP9, MMP3, MMP13, TIMP3, MMP2, MMP12, MMP1, TIMP1 \\
Osteoarthritis & $1.99 \mathrm{E}-05$ & MATN3, PTGS2, MATN1, SERPINA3, IL1B, COL2A1, MMP2 \\
Nasopharyngeal cancer & $2.29 \mathrm{E}-06$ & MMP9, MMP7, MMP3, MMP13, MMP2, MMP12, MMP1 \\
Chronic obstructive pulmonary disease & $2.22 \mathrm{E}-05$ & MMP9, SERPINA3, IL1B, SERPINA1, MMP14, MMP12, MMP1 \\
\hline
\end{tabular}

\section{DISCUSSION}

In this study, we investigated the association between serum levels of ADAMTS-4, ADAMTS-5, MMP-1, and MMP-3 and the severity of OA. We found that the ADAMTS-4 levels in early OA patients were significantly higher than those in intermediate and advanced OA patients and healthy controls, whereas ADAMTS-5, MMP-1, and MMP-3 levels in intermediate and advanced OA patients were significantly higher than those in early OA patients and healthy controls.

A large number of studies have recently been conducted to examine the ADAMTS family. In general, ADAMTS-5 is constitutively expressed in human chondrocytes and synovial fibroblasts, whereas ADAMTS-4 expression is induced by proinflammatory cytokines (Fosang et al., 2008). The active forms of ADAMTS-4 were increased in synovial fluid samples from patients with active Lyme arthritis (Behera et al., 2006). Expression of ADAMTS-4 in the surface zone of human osteoarthritic cartilage is regulated by epigenetic DNA demethylation (Cheung et al., 2009). Suppression of ADAMTS-4 and ADAMTS-5, individually or in combination, attenuated the degradation of aggrecan in cytokine-stimulated normal cartilage (Song et al., 2007). Mice with a double-knockout of ADAMTS-4 and ADAMTS-5 were physiologically normal animals in which OA was prevented (Majumdar et al., 2007). $\mathrm{a}_{2}$-Macroglobulin is an endogenous inhibitor of ADAMTS-4 and ADAMTS-5 (Tortorella et al., 2004). Fibroblast growth factor 2 is an intrinsic chondroprotective agent that suppresses ADAMTS-5 and delays cartilage degradation in murine OA (Chia et al., 2009). Our results suggest that ADAMTS-4 plays a leading role in the early stage of OA, while ADAMTS-5 affects intermediate and advanced stages of OA.

MMP-1 and MMP-3 levels are reportedly correlated in synovial fluid in rheumatoid arthritis and OA (Tchetverikov et al., 2004). MMP-1 and MMP-3 levels were also consistently expressed in the serum in our study. MMP-1 activity was increased in the synovial fluid of OA joints in horses (Brama et al., 2004). Overexpression of serum amyloid A-activating factor 1 in OA chondrocytes increased the expression of the MMP-1 promoter; thus, serum amyloid A-activating factor 1 may be a therapeutic target for controlling the overexpression of MMP-1 in OA (Ray et al., 2003). Increased MMP-3 can induce matrix degradation of articular cartilage in mature bovines (Lin et al., 2004). The serum concentration of MMP-3 is also a useful marker for predicting bone damage in the early stage of rheumatoid arthritis (Yamanaka et al., 2000). It was reported that serum MMP-3 levels did not differ between OA patients and normal sera; however, these patients were not assessed based on a grading system (Mahmoud et al., 2005). Our results suggest that MMP-1 and MMP-3 affect OA mainly during intermediate and advanced stages. 
The PPI network indicated that a complicated interaction exists between ADAMTS-4 and other proteins, and that the genes encoding those proteins were associated with rheumatoid arthritis and OA.

In conclusion, we confirmed that ADAMTS-4 levels in early OA patients were significantly higher than in intermediate and advanced OA patients and healthy controls, making it a potential biomarker as an early diagnostic indicator of OA. However, because of the complex interaction with other proteins, the results should be further examined.

\section{Conflicts of interest}

We declare that we have no conflict of interest.

\section{ACKNOWLEDGMENTS}

Research supported by the Science and Technology Key Projects of the Department of Science \& Technology of Shandong Province, China (\#2008GGB14823, \#2013GGB14067).

\section{REFERENCES}

Ameye LG and Young MF (2006). Animal models of osteoarthritis: lessons learned while seeking the 'Holy Grail'. Curr. Opin. Rheumatol. 18: 537-547.

Arendt-Nielsen L, Nie H, Laursen MB, Laursen BS, et al. (2010). Sensitization in patients with painful knee osteoarthritis. Pain 149: 573-581.

Behera AK, Hildebrand E, Szafranski J, Hung HH, et al. (2006). Role of aggrecanase 1 in Lyme arthritis. Arthritis Rheum. 54: 3319-3329.

Bijlsma JW, Berenbaum F and Lafeber FP (2011). Osteoarthritis: an update with relevance for clinical practice. Lancet 377: $2115-2126$.

Brama PA, van den Boom R, DeGroot J, Kiers GH, et al. (2004). Collagenase-1 (MMP-1) activity in equine synovial fluid: influence of age, joint pathology, exercise and repeated arthrocentesis. Equine Vet. J. 36: 34-40.

Cheung KS, Hashimoto K, Yamada N and Roach HI (2009). Expression of ADAMTS-4 by chondrocytes in the surface zone of human osteoarthritic cartilage is regulated by epigenetic DNA de-methylation. Rheumatol. Int. 29: 525-534.

Chia SL, Sawaji Y, Burleigh A, McLean C, et al. (2009). Fibroblast growth factor 2 is an intrinsic chondroprotective agent that suppresses ADAMTS-5 and delays cartilage degradation in murine osteoarthritis. Arthritis Rheum. 60: 2019-2027.

Fosang AJ, Rogerson FM, East CJ and Stanton H (2008). ADAMTS-5: the story so far. Eur. Cell Mater. 15: 11-26.

Glasson SS, Askew R, Sheppard B, Carito BA, et al. (2004). Characterization of and osteoarthritis susceptibility in ADAMTS-4-knockout mice. Arthritis Rheum. 50: 2547-2558.

Huang da W, Sherman BT and Lempicki RA (2009). Systematic and integrative analysis of large gene lists using DAVID bioinformatics resources. Nat. Protoc. 4: 44-57.

Kapoor M, Martel-Pelletier J, Lajeunesse D, Pelletier JP, et al. (2011). Role of proinflammatory cytokines in the pathophysiology of osteoarthritis. Nat. Rev. Rheumatol. 7: 33-42.

Lin PM, Chen CT and Torzilli PA (2004). Increased stromelysin-1 (MMP-3), proteoglycan degradation (3B3-and 7D4) and collagen damage in cyclically load-injured articular cartilage. Osteoarthritis Cartilage 12: 485-496.

Mahmoud RK, El-Ansary AK, H. El-Eishi HH, Kamal HM, et al. (2005). Matrix metalloproteinases MMP-3 and MMP-1 levels sera and synovial fluids in patients with rheumatoid arthritis and osteoarthritis. Ital. J. Biochem. 54: 248-257.

Majumdar MK, Askew R, Schelling S, Stedman N, et al. (2007). Double-knockout of ADAMTS-4 and ADAMTS-5 in mice results in physiologically normal animals and prevents the progression of osteoarthritis. Arthritis Rheum. 56: 3670-3674.

Mizui Y, Yamazaki K, Kuboi Y, Sagane K, et al. (2000). Characterization of $5 \phi$-flanking region of human aggrecanase-1 (ADAMTS4) gene. Mol. Biol. Rep. 27: 167-173.

Poole AR, Ionescu M, Swan A and Dieppe P (1994). Changes in cartilage metabolism in arthritis are reflected by altered serum and synovial fluid levels of the cartilage proteoglycan aggrecan. Implications for pathogenesis. J. Clin. Invest. 
94: 25-33.

Porter S, Clark IM, Kevorkian L and Edwards DR (2005). The ADAMTS metalloproteinases. Biochem. J. 386: 15-27.

Ray A, Kuroki K, Cook JL, Bal BS, et al. (2003). Induction of matrix metalloproteinase 1 gene expression is regulated by inflammation-responsive transcription factor SAF-1 in osteoarthritis. Arthritis Rheum. 48: 134-145.

Recht MP, Kramer J, Marcelis S, Pathria MN, et al. (1993). Abnormalities of articular cartilage in the knee: analysis of available MR techniques. Radiology 187: 473-478.

Song RH, Tortorella MD, Malfait AM, Alston JT, et al. (2007). Aggrecan degradation in human articular cartilage explants is mediated by both ADAMTS-4 and ADAMTS-5. Arthritis Rheum. 56: 575-585.

Takahashi K, Goomer RS, Harwood F, Kubo T, et al. (1999). The effects of hyaluronan on matrix metalloproteinase-3 (MMP-3), interleukin-1 $\beta$ (IL-1b), and tissue inhibitor of metalloproteinase-1 (TIMP-1) gene expression during the development of osteoarthritis. Osteoarthritis Cartilage 7: 182-190.

Tchetverikov I, Ronday HK, Van El B, Kiers GH, et al. (2004). MMP profile in paired serum and synovial fluid samples of patients with rheumatoid arthritis. Ann. Rheum. Dis. 63: 881-883.

Tortorella MD, Arner EC, Hills R, Easton A, et al. (2004). Alpha2-macroglobulin is a novel substrate for ADAMTS-4 and ADAMTS-5 and represents an endogenous inhibitor of these enzymes. J. Biol. Chem. 279: 17554-17561.

Verma P and Dalal K (2011). ADAMTS-4 and ADAMTS-5: Key enzymes in osteoarthritis. J. Cell Biochem. 112: $3507-$ 3514.

Wei M, Duan D, Liu Y, Wang Z, et al. (2013). Increased thymosin beta4 levels in the serum and SF of knee osteoarthritis patients correlate with disease severity. Regul. Pept. 185: 34-36.

Yamanaka H, Matsuda Y, Tanaka M, Sendo W, et al. (2000). Serum matrix metalloproteinase 3 as a predictor of the degree of joint destruction during the six months after measurement, in patients with early rheumatoid arthritis. Arthritis Rheum. 43: 852-858.

Yoshihara Y, Nakamura H, Obata K, Yamada H, et al. (2000). Matrix metalloproteinases and tissue inhibitors of metalloproteinases in synovial fluids from patients with rheumatoid arthritis or osteoarthritis. Ann. Rheum. Dis. 59: 455-461.

Zeggini E, Panoutsopoulou K, arcOGEN Consortium, arcORGEN Collaborators, et al. (2012). Identification of new susceptibility loci for osteoarthritis (arcOGEN): a genome-wide association study. Lancet 380: 815-823. 